\title{
The generalized Ackermann's formula for singular systems
}

\author{
Lee-Chuang Hsu, Fan-Ren Chang* \\ Department of Electrical Engineering, Rm 241, National Taiwan University, Taipei, Taiwan 10617, ROC \\ Received 15 April 1995; revised 15 November 1995
}

\begin{abstract}
Being an elegant algorithm for state feedback pole placement, Ackermann's (1972) formula had been widely quoted in control texts. In this paper, the formula is extended to solve the root assignment problem for singular systems. Without loss of generality, it is both well known and convenient that any regular generalized system can be transformed into the standard form $E \dot{x}=A x(t)+b u(t)$, where $\mu E-A=I$ and $\mu$ is a real constant. In the derivation of the generalized Ackermann's formule, the closed-loop characteristic polynomial, $\operatorname{det}\left[s E-A+b k^{\prime}\right]$, is simplified due to the relationship of $E$ and $A$. If $E$ is nonsingular, the feedback gain $k^{\prime}$ can be computed from the generalized Ackermann's formula directly. In this case, only the desired closed-loop characteristic polynomial is required. If $E$ is singular, the feedback algorithm needs both closed-loop and open-loop characteristic polynomials. Two numerical examples are presented to demonstrate our algorithms.
\end{abstract}

Keywords: Generalized state-space systems; Descriptor systems; Pole placement; State feedback; Ackermann's formula

\section{Introduction}

In this paper, we consider the single input singular (or generalized, descriptor, semi-state) linear system

$\bar{E} \dot{x}(t)=\bar{A} x(t)+\bar{b} u(t)$

where $\bar{E}$ and $\bar{A} \in \mathbb{R}^{n \times n}, \bar{b} \in \mathbb{R}^{n \times 1}, \bar{E}$ is possibly a singular matrix, and $u(t) \in \mathbb{R}$ and $x(t) \in \mathbb{R}^{n}$ are input and state vectors respectively. The problem of pole placement in singular systems is to find the state feedback control law $u(t)=-k^{\prime} x(t)+r(t)$, where $k^{\prime} \in \mathbb{R}^{1 \times n}$ and $r(t) \in \mathbb{R}$ such that the closed-loop system has the prescribed finite and infinite poles.

The problems of pole placement in generalized state-space systems have been treated in many pa-

\footnotetext{
* Corresponding author.
}

pers. In [6], the decomposed fast and slow subsystems were used to assign finite poles and eliminate impulsive motions. The same form was employed in [3] to show that the controllability of infinite poles is equivalent to the existence of a state feedback map which shifts those poles to specified complex numbers. By applying a special coordinate transformation, pole placement and compensator design were considered in [15]. For more details about this problem, please see [2] and the reference cited therein.

In this paper, we solve the pole placement problem by extending the famous Ackermann's formula $[5,8,10,12]$ from state-space systems $\dot{x}(t)=$ $A x(t)+b u(t)$ to standard singular systems. In Section 2 , we quickly review the restricted system equivalent. Under a very simple restricted equivalent transformation, (1) can be converted to standard forms $E \dot{x}(t)=A x(t)+b u(t)$. With the 
preliminary results of Section 2, we can develop the main theorems in Section 3. The generalized Ackermann's formula for standard singular systems is established in Theorem 1. The pole placement feedback gain $k^{\prime}$ can be obtained from Theorem 1 if $E$ is nonsingular. To compute $k^{\prime}$ for the case of singular $E$, Theorem 2 is proposed. Theorem 1 only needs closed-loop characteristic polynomials. Theorem 2, however, needs both open-loop and closed-loop characteristic polynomials. To demonstrate our results, two numerical examples are presented in Section 4. Brief conclusions are given in Section 5 .

\section{Preliminaries}

Definition 1 (Gantmacher [9]). Let $\bar{E}$ and $\bar{A}$ be two square constant matrices. If $\operatorname{det}(s \bar{E}-\bar{A}) \lessgtr 0$ then $s \bar{E}-\bar{A}$ is a regular pencil

If $\bar{E}$ and $\bar{A}$ form a regular pencil, then $\bar{E} \dot{x}(t)=$ $\bar{A} x(t)+\bar{b} u(t)$ is a regular generalized system.

Definition 2 (Nikoukhan et al. [11]). Let $s E-A$ be a regular pencil. If there exist scalars $\alpha$ and $\beta$ such that $\alpha E+\beta A=I$, where $I$ is the identity matrix, then $s E-A$ is a standard pencil.

If $E$ and $A$ form a standard pencil, then $E \dot{x}(t)=$ $A x(t)+b u(t)$ is a standard generalized system.

Definition 3 (Rosenbrock [13]). For the system (1), and the system (2) given by

$E \dot{x}(t)=A x(t)+b u(t)$

if there exist two nonsingular matrices $M, N \in \mathbb{R}^{n \times n}$ such that

$E=M \bar{E} N, \quad A=M \bar{A} N, \quad b=M \bar{b}$

then (1) and (2) are restricted system equivalent.

Any regular generalized system (1) can be converted to a standard generalized system by a restricted system equivalent transformation. By letting a scalar $\mu$ satisfying $\operatorname{det}(\mu \bar{E}-\bar{A}) \neq 0$, we select $M=(\mu \bar{E}-\bar{A})^{-1}$ and $N=I$. The results of the transformation are

$$
\begin{aligned}
& E=(\mu \bar{E}-\bar{A})^{-1} \bar{E}, \\
& A=(\mu \bar{E}-\bar{A})^{-1} \bar{A}, \\
& b=(\mu \bar{E}-\bar{A})^{-1} \bar{b} .
\end{aligned}
$$

From (3a) and (3b), it is easy to verify that

$\mu E-A=I$

For matrices $E$ and $A$ in (4), $s E-A$ is a standard pencil, since $\alpha=\mu$ and $\beta=-1$. Hence, the restricted equivalent system (2) is a standard generalized system. Henceforth, we shall assume the matrices $E$ and $A$ always satisfy (4).

Two restricted equivalent systems possess the same finite and infinite modes [14]. The characteristic polynomials of (1) and (2) are different in a constant multiplier.

$$
\begin{aligned}
\operatorname{det}(s E-A) & =\operatorname{det}\left[(\mu \bar{E}-\bar{A})^{-1}\right] \operatorname{det}(s \bar{E}-\bar{A}) \\
& =c \cdot \operatorname{det}(s \bar{E}-\bar{A})
\end{aligned}
$$

where $c=\operatorname{det}\left[(\mu \bar{E}-\bar{A})^{-1}\right]$, a nonzero constant.

In the following, we summarize the controllabilities of generalized systems.

Lemma 1 (Cobb [7]). The generalized system (1) is controllable if and only if

$\operatorname{rank}[s \bar{E}-\bar{A} \bar{b}]=n$ for all finite $s$

and

$\operatorname{rank}\left[\begin{array}{ll}\bar{E} & \bar{b}\end{array}\right]=n$.

Lemma 2 (Cobb [6]). The standard generalized system (2) is controllable if and only if

$\operatorname{rank}\left[\begin{array}{lllll}b & E b & E^{2} b & \cdots & E^{n-1} b\end{array}\right]=n$.

Note that the restricted equivalence preserves the controllabilities also.

Definition 4. The finite eigenvalues of a regular pencil $s E-A$ are the roots of the characteristic polynomials $\operatorname{det}(s \bar{E}-\bar{A})$.

Lemma 3. If $p_{i}$ are nonzero eigenvalues of $E$, then $s_{i} \equiv \mu-1 / p_{i}$ are finite eigenvalues of the standard pencil $s E-A$.

Proof. $p_{i}$ are eigenvalues of $E, \operatorname{det}\left(p_{i} I-E\right)=0$. Also, $\quad \operatorname{det}\left(p_{i} I-E\right)=\operatorname{det}\left[p_{i}(\mu E-A)-E\right]=$ $\left(p_{i}\right)^{n} \operatorname{det}\left[\left(\mu_{i}-1 / p_{i}\right) E-A\right]$. Since $p_{i}$ are nonzero, we have $\operatorname{det}\left[\left(\mu-1 / p_{i}\right) E-A\right]=0$. Hence $s_{i} \equiv$ $\left(\mu-1 / p_{i}\right)$ are finite eigenvalues of $s E-A$.

Lemma 4. If $p_{i}$ are zero eigenvalues of $E$, then the standard pencil $(s E-A)$ has infinite eigenvalues. 
Proof. If $E$ contains zero eigenvalues, then $\operatorname{det} E=0$. Also, $(s E-A)$ has infinite eigenvalues if and only if the pencil $(\lambda A-E)$ has zero eigenvalues.

$\left.\operatorname{det}(\lambda A-E)\right|_{\lambda=0}=\operatorname{det}(-E)=0$.

From Lemmas 3 and 4 , the finite and infinite eigenvalues of the s:andard pencil $s E-A$ can be expressed in terms of eigenvalues of $E, p_{i}$.

Lemma 5. For the case of $\mu E-A=I$ and $s \neq \mu$,

$\operatorname{det}(s E-A)=\left(\frac{1}{p}\right)^{n} \operatorname{det}(p I-E)$

$\operatorname{det}\left(s E-\left(A-b k^{\prime}\right)\right]:=\left(\frac{1}{p}\right)^{n} \operatorname{det}\left[p\left(I+b k^{\prime}\right)-E\right]$,

where $p=1 /(\mu-s)$ or $s=\mu-1 / p$.

Proof. Substitute $A=\mu E-I$. The result follows.

From [4], it is easy to show Lemma 6.

Lemma 6. For the standard pencil $s E-A$ with $\mu E-A=I$, if

$$
\begin{aligned}
\Delta_{o}(p) & =\left.(p)^{n} \operatorname{det}(s E-A)\right|_{s=\mu-1 / p, p \neq 0}=\operatorname{det}(p I-E) \\
& =\sum_{i=0}^{n} a_{n-i} p^{i},
\end{aligned}
$$

with $a_{0}=1$, then $\Delta_{0}(E)=\sum_{i=0}^{n} a_{n-i} E^{i}=0$.

There are two matrices ( $E$ and $A$ ) involved in the open-loop characteristic polynomial in the lefthand side of (9). However, there is only one matrix $(E)$ involved for the equivalent information in the right-hand side of the same equation. Also, the closed-loop characteristic polynomial in the righthand side of (10) is simpler than in the left-hand side.

\section{Main results}

With the help of previous lemmas, two theorems will be developed in this section. Theorem 1 can be viewed as the Ackermann's formula in generalized state-space systems. Theorem 2 provides a computational algorithrn for state feedback when $E$ is singular.
Theorem 1 (Generalized Ackermann's formula). Let $E \dot{x}(t)=A x(t)+b u(t)$ be a standard controllable generalized system, satisfying $\mu E-A=I$. Assume the state feedback control law is $u(t)=-k^{\prime} x(t)+$ $r(t)$ and the desired closed-loop characteristic polynomial is

$$
\begin{aligned}
\Delta_{d}(p) & =\left.(p)^{n} \operatorname{det}\left[s E-\left(A-b k^{\prime}\right)\right]\right|_{s=\mu-1 / p} \\
& =\sum_{i=0}^{n} d_{n-i} p^{i},
\end{aligned}
$$

where $d_{n}=\operatorname{det}(-E)$. Then

$k^{\prime} E=e_{n}^{\prime} C^{-1} \Delta_{d}(E)$

where

$$
\begin{aligned}
& e_{n}^{\prime}=\left[\begin{array}{lllll}
0 & 0 & \cdots & 0 & 1
\end{array}\right], \\
& C=\left[\begin{array}{llll}
b & E b & \cdots & E^{n-1} b
\end{array}\right], \\
& \Delta_{d}(E)=\sum_{i=0}^{n} d_{n-i} E^{i}
\end{aligned}
$$

Proof. By Lemma $2 \operatorname{rank} C=n .\{E, b\}$ thus can be transformed to its controllable canonical form $\left\{E_{\mathrm{c}}, b_{\mathrm{c}}\right\}[10]$.

$E_{\mathrm{c}}=T E T^{-1}$

$$
=\left[\begin{array}{ccccc}
0 & 0 & 0 & \cdots & 0 \\
0 & 0 & 1 & \cdots & 0 \\
0 & 0 & 0 & \cdots & 1 \\
-a_{n} & -a_{n-1} & -a_{n-2} & \cdots & -a_{1}
\end{array}\right],
$$

$b_{\mathrm{c}}=T b=\left[\begin{array}{c}0 \\ 0 \\ \vdots \\ 0 \\ 1\end{array}\right]$,

where

$$
T=\left[\begin{array}{c}
q^{\prime} \\
q^{\prime} E \\
q^{\prime} E^{2} \\
\vdots \\
q^{\prime} E^{n-1}
\end{array}\right]
$$

$q^{\prime} \equiv$ the last row of the $C^{-1}=e_{n}^{\prime} C^{-1}$.

From (13a), $\operatorname{det}\left(p I-E_{\mathrm{c}}\right)=a_{n}+a_{n-1} p+a_{n-2} p^{2}$ $+\cdots+a_{1} p^{n-1}+p^{n}$, where $a_{n}=\operatorname{det}\left(-E_{\mathrm{c}}\right)$. By (9) 
in Lemma 5, the open-loop characteristic polynomials expressed in $s(=\mu-1 / p)$ and $p(=1 /(\mu-s))$ are related by

$$
\begin{aligned}
p^{n} \operatorname{det}(s E-A)= & p^{n} \operatorname{det}\left(s E_{\mathrm{c}}-A_{\mathrm{c}}\right)=\operatorname{det}(p I-E) \\
= & \operatorname{det}\left(p I-E_{\mathrm{c}}\right)=\Delta_{o}(p) \\
= & a_{n}+a_{n-1} p+a_{n-2} p^{2} \\
& +\cdots+a_{1} p^{n-1}+p^{n}
\end{aligned}
$$

where $A_{\mathrm{c}}=T A T^{-1}$. By (10) in Lemma 5, the closed-loop characteristic polynomials expressed in $s(=\mu-1 / p)$ and $p(=1 /(\mu-s))$ are related by

$$
\begin{aligned}
p^{n} \operatorname{det}\left(s E-A+b k^{\prime}\right) & =p^{n} \operatorname{det}\left(s E_{\mathrm{c}}-A_{\mathrm{c}}+b_{\mathrm{c}} k_{\mathrm{c}}^{\prime}\right) \\
& =\operatorname{det}\left[p\left(I+b k^{\prime}\right)-E\right] \\
& =\operatorname{det}\left[p\left(I+b_{\mathrm{c}} k_{\mathrm{c}}^{\prime}\right)-E_{\mathrm{c}}\right]
\end{aligned}
$$

where $k_{\mathrm{c}}^{\prime}=k^{\prime} T^{-1}$. Assume

$k_{\mathrm{c}}^{\prime}=\left[\begin{array}{lllll}k_{n} & k_{n-1} & k_{n-2} & \cdots & k_{1}\end{array}\right]$

Then by direct multiplication,

$$
\begin{aligned}
\operatorname{det}\left[p\left(I+b_{\mathrm{c}} k_{\mathrm{c}}^{\prime}\right)-E_{\mathrm{c}}\right]= & a_{n}+\left(a_{n-1}+k_{n}\right) p \\
& +\left(a_{n-2}+k_{n-1}\right) p^{2} \\
& +\cdots+\left(a_{1}+k_{2}\right) p^{n-1} \\
& +\left(1+k_{1}\right) p^{n} .
\end{aligned}
$$

The desired closed-loop characteristic polynomial is

$$
\begin{aligned}
\Delta_{d}(p)= & d_{n}+d_{n-1} p+d_{n-2} p^{2} \\
& +\cdots+d_{1} p^{n-1}+d_{0} p^{n}
\end{aligned}
$$

where $d_{n}=\operatorname{det}(-E)=a_{n}$. Comparing (16) and (17), we have

$$
\begin{aligned}
& k_{\mathrm{c}}^{\prime}=\left[\begin{array}{llll}
d_{n-1}-a_{n-1} & d_{n-2}-a_{n-2} & \cdots & d_{1}-a_{1}
\end{array}\right. \\
& \left.d_{0}-1\right] \text {. }
\end{aligned}
$$

In order to obtain Ackermann's formula, let us examine the left side of (11)

$$
\begin{aligned}
k^{\prime} E= & k_{\mathrm{c}}^{\prime} T E \\
= & {\left[\begin{array}{llll}
d_{n-1}-a_{n-1} & d_{n-2}-a_{n-2} & \cdots & d_{1}-a_{1} d_{0}-1
\end{array}\right] } \\
& \times\left[\begin{array}{c}
q^{\prime} E \\
q^{\prime} E^{2} \\
\vdots \\
q^{\prime} E^{n}
\end{array}\right]
\end{aligned}
$$

$$
\begin{aligned}
= & q^{\prime}\left(d_{n-1} E+d_{n-2} E^{2}+\cdots+d_{1} E^{n-1}+d_{0} E^{n}\right) \\
& -q^{\prime}\left(a_{n-1} E+a_{n-2} E^{2}+\cdots+a_{1} E^{n-1}+E^{n}\right) \\
= & q^{\prime}\left(d_{n} I+d_{n-1} E+\cdots+d_{1} E^{n-1}+d_{0} E^{n}\right) \\
& -q^{\prime}\left(a_{n} I+a_{n-1} E+a_{n-2} E^{2}\right. \\
& \left.\quad+\cdots+a_{1} E^{n-1}+E^{n}\right) \\
& \quad\left(\because d_{n}=a_{n}=\operatorname{det}(-E)\right) \\
= & q^{\prime} \Delta_{d}(E)-q^{\prime} \Delta_{o}(E) \\
= & e_{n}^{\prime} C^{-1} \Delta_{d}(E)
\end{aligned}
$$

since $q^{\prime}=e_{n}^{\prime} C^{-1}$ and $\Delta_{o}(E)=0$ by Lemma 6 .

Remark 1. If $\bar{E} \dot{x}(t)=\bar{A} x(t)+\bar{b} u(t)$ is controllable but not necessarily standard, then we just left multiply both sides of the equation by $(\mu \bar{E}-\bar{A})^{-1}$. In doing so, we change neither state vector $x(t)$ nor input $u(t)$. However, the obtained $E \dot{x}(t)=$ $A x(t)+b u(t)$, where $E, A$ and $b$ are shown in (3), becomes a standard controllable singular system. Then the generalized Ackermann's formula can be applied. Furthermore, if the gain $k^{\prime}$ in (11) is used in the original system for state feedback, then the closed-loop characteristic polynomial will be $\operatorname{det}\left(s \bar{E}-\bar{A}+\bar{b} k^{\prime}\right)=c \cdot \operatorname{det}\left(s E-A+b k^{\prime}\right)$ with $c \equiv$ $\operatorname{det}(\mu \bar{E}-\bar{A})$, a nonzero constant. In other words, the closed-loop finite poles are the same desired set.

Remark 2. In the desired closed-loop characteristic polynomial $\Delta_{d}(p)=\sum_{i=0}^{n} d_{n-i} p^{i}$, there are $n$ coefficients $d_{n-1}, d_{n-2}, \ldots, d_{0}$ which can be arbitrarily assigned. The constant term $d_{n}$ is fixed and is equal to $\operatorname{det}(-E)$.

Remark 3. If the matrix $E$ is nonsingular, then there are $n$ arbitrary roots, $p_{i}$, which can be selected by the appropriate choice of $d_{1}, d_{2}, \ldots, d_{n}$. The associated closed-loop eigenvalues of $\left(s E-A+b k^{\prime}\right)$ are $s_{i}=\mu-1 / p_{i}$, for $p_{i} \neq 0$. If $p_{i}=0$, then they will be infinite poles for closed-loop polynomials. Also, the feedback gain $k^{\prime}$ will be

$k^{\prime}=e_{n}^{\prime} C^{-1} \Delta_{d}(E) E^{-1}$

where $E$ is nonsingular.

Remark 4. Let $\Delta_{d}(p)=\sum_{i=1}^{n} d_{n-i} p^{i}$ be the desired closed-loop characteristic polynomial. From Remark $2, d_{n}=\operatorname{det}(-E)$. If $E$ is singular, then $d_{n}=0$. In this case, we see that at least one root of $\Delta_{d}(p)$ is 0 . This means that at least one infinite pole 
remains in the closed-loop system. This result is coincident with well-known state feedback properties of controllable generalized systems.

Remark 5. If $E$ is singular, then (19) cannot be applied. Hence we will develop Theorem 2 to compute feedback gain $k^{\prime}$.

Theorem 2. Let $E \dot{x}(t)=A x(t)+b u(t)$, with $E$ singular, be a standard controllable generalized system, satisfying $\mu E-A=I$. Assume the desired closed-loop characteristic polynomial is $\Delta_{d}(p)=$ $\left.(p)^{n} \operatorname{det}\left[s E-\left(A-b k^{\prime}\right)\right]\right|_{s=\mu-1 / p}=\sum_{i=1}^{n} d_{n-i} p^{i}$. Then

$$
\begin{aligned}
k^{\prime}= & e_{n}^{\prime} C^{-1}\left[\left(d_{n-1}-a_{n-1}\right) I+\left(d_{n-2}-a_{n-2}\right) E\right. \\
& \left.+\cdots+\left(d_{1}-a_{1}\right) E^{n-2}+\left(d_{0}-1\right) E^{n-1}\right]
\end{aligned}
$$

where

$$
\begin{aligned}
& e_{n}^{\prime}=\left[\begin{array}{lllll}
0 & 0 & \cdots & 0 & 1
\end{array}\right], \\
& C=\left[\begin{array}{llll}
b & E b & \cdots & E^{n-1} b
\end{array}\right], \\
& \operatorname{det}(p I-E)=\sum_{i=1}^{n-1} a_{n-i} p^{i}+p^{n} .
\end{aligned}
$$

\section{Proof}

$$
\begin{aligned}
k^{\prime}= & k_{\mathrm{c}}^{\prime} T \\
= & {\left[d_{n-1}-a_{n-1} d_{n-2}-a_{n-2} \cdots d_{1}-a_{1} d_{0}-1\right] } \\
& \times\left[\begin{array}{c}
q^{\prime} \\
q^{\prime} E \\
\vdots \\
q^{\prime} E^{n-1}
\end{array}\right] \\
= & q^{\prime}\left[\left(d_{n-1}-a_{n-1}\right) I+\left(d_{n-2}-a_{n-2}\right) E\right. \\
& \left.+\cdots+\left(d_{1}-a_{1}\right) E^{n-2}+\left(d_{0}-1\right) E^{n-1}\right]
\end{aligned}
$$

also $q^{\prime}=e_{n}^{\prime} C^{-1}$.

Remark 6. (21c) is the characteristic polynomial of $E$. It is equivalent to an open-loop characteristic polynomial.

\section{Illustrative examples}

Two illustrative examples are presented step by step in this section.
Example 1 ( $\bar{E}$ is nonsingular). Consider a generalized state-space system as (1), given as follows:

$\bar{E}=\left[\begin{array}{lll}1 & 1 & 0 \\ 0 & 0 & 1 \\ 0 & 1 & 0\end{array}\right], \quad \bar{A}=\left[\begin{array}{lll}1 & 0 & 0 \\ 0 & 1 & 0 \\ 0 & 0 & 1\end{array}\right]$

and

$\bar{b}=\left[\begin{array}{l}1 \\ 0 \\ 1\end{array}\right]$

Let the desired eigenvalues of the closed-loop be $s_{1 d}=-1, s_{2 d}=-1, s_{3 d}=-2$. Find the state feedback gain $k^{\prime}$.

\section{Solution}

Step 1: Convert the regular pencil to the standard pencil. Set $\mu=0$

$$
\begin{aligned}
& E=(\mu \bar{E}-\bar{A})^{-1} \bar{E}=\left[\begin{array}{rrr}
-1 & -1 & 0 \\
0 & 0 & -1 \\
0 & -1 & 0
\end{array}\right], \\
& A=(\mu \bar{E}-\bar{A})^{-1} \bar{A}=\left[\begin{array}{rrr}
-1 & 0 & 0 \\
0 & -1 & 0 \\
0 & 0 & -1
\end{array}\right], \\
& b=(\mu \bar{E}-\bar{A})^{-1} \bar{b}=\left[\begin{array}{r}
-1 \\
0 \\
-1
\end{array}\right] .
\end{aligned}
$$

Step 2: The closed-loop poles, in terms of $p$, are converted by $p_{i d}=1 /\left(\mu-s_{i d}\right)$. Closed-loop poles: $s_{1 d}=s_{2 d}=-1 \quad$ and $\quad s_{3 d}=-2 \Rightarrow p_{1 d}=p_{2 d}=1$ and $p_{3 d}=\frac{1}{2}$. Hence the desired closed-loop characteristic polynomial, in terms of $p$, is $\Delta_{d}(p)=$ $r\left(p-p_{1 d}\right)\left(p-p_{2 d}\right)\left(p-p_{3 d}\right)=r\left(p^{3}-2.5 p^{2}+\right.$ $2 p-0.5)=d_{0} p^{3}+d_{1} p^{2}+d_{2} p+d_{3}$ in which $r$ is the scaling factor such that $d_{3}=\operatorname{det}(-E)=-1$. From $r(-0.5)=d_{3}=-1$, we see that $r=2$ and $\Delta_{d}(p)=2 p^{3}-5 p^{2}+4 p-1$.

Step 3: Find the feedback gain $k^{\prime}$ by (19).

$$
k^{\prime}=e_{3}^{\prime} C^{-1} \Delta_{d}(E) E^{-1}=\left[\begin{array}{lll}
-12 & 5 & 11
\end{array}\right]
$$

where

$$
C^{-1}=\left[\begin{array}{lll}
b & E b & E^{2} b
\end{array}\right]^{-1}=\left[\begin{array}{rrr}
1 & -1 & -2 \\
0 & 1 & 0 \\
-1 & 1 & 1
\end{array}\right]
$$


Example 2 ( $\bar{E}$ is singular). Consider a generalized state-space system as (1), given as follows:

$\bar{E}=\left[\begin{array}{ccc}0 & 0 & 0 \\ 0 & 1 & 1 \\ 1 & 0 & 0\end{array}\right], \quad \bar{A}=\left[\begin{array}{ccc}1 & 0 & 0 \\ 0 & 2 & 0 \\ 0 & 0 & 1\end{array}\right]$

and

$\bar{b}=\left[\begin{array}{l}1 \\ 0 \\ 0\end{array}\right]$.

Let the desired eigenvalues of the closed-loop be $s_{1 d}=\infty, s_{2 d}=-1$ and $s_{3 d}=-2$. Find the state feedback gain $k^{\prime}$.

\section{Solution}

Step 1: Convert the regular pencil to the standard pencil. Set $\mu=0$

$E=(\mu \bar{E}-\bar{A})^{-1} \bar{E}=\left[\begin{array}{ccc}0 & 0 & 0 \\ 0 & -0.5 & -0.5 \\ -1 & 0 & 0\end{array}\right]$,

$A=(\mu \bar{E}-\bar{A})^{-1} \bar{A}=\left[\begin{array}{rrr}-1 & 0 & 0 \\ 0 & -1 & 0 \\ 0 & 0 & -1\end{array}\right]$,

$b=(\mu \bar{E}-\bar{A})^{-1} \bar{b}=\left[\begin{array}{r}-1 \\ 0 \\ 0\end{array}\right]$.

Step 2: The open-loop poles, in terms of $p$, are eigenvalues of $E$. The closed-loop poles, in terms of $p$, are converted by $p_{i d}=1 /\left(\mu-s_{i d}\right)$

Open-loop poles: eigenvalues of $E$ are

$$
p_{1}=p_{2}=0 \text { and } p_{3}=-\frac{1}{2} \text {. }
$$

Closed-loop poles: $s_{1 d}=\infty, s_{2 d}=-1$ and

$$
\begin{aligned}
& s_{3 d}=-2 \Rightarrow p_{1 d}=0, p_{2 d}=1 \\
& \text { and } p_{3 d}=\frac{1}{2} .
\end{aligned}
$$

Hence we have that the characteristic polynomials, in terms of $p$, are

Open-loop characteristic polynomial:

$$
\begin{aligned}
\Delta_{o}(p) & =\left(p-p_{1}\right)\left(p-p_{2}\right)\left(p-p_{3}\right) \\
& =p^{3}+a_{1} p^{2}+a_{2} p+a_{3}=p^{3}+0.5 p^{2} .
\end{aligned}
$$

Closed-loop characteristic polynomial:

$$
\begin{aligned}
\Delta_{d}(p) & =\left(p-p_{1 d}\right)\left(p-p_{2 d}\right)\left(p-p_{3 d}\right) \\
& =d_{0} p^{3}+d_{1} p^{2}+d_{2} p+d_{3}=p^{3}-1.5 p^{2}+0.5 p .
\end{aligned}
$$

Step 3: Find the feedback gain $k^{\prime}$ by Theorem 2

$$
\begin{aligned}
k^{\prime} & =e_{3}^{\prime} C^{-1}\left[\left(d_{2}-a_{2}\right) I+\left(d_{1}-a_{1}\right) E+\left(d_{0}-1\right) E^{2}\right] \\
& =\left[\begin{array}{lll}
0 & -3 & -2
\end{array}\right]
\end{aligned}
$$

where

$C^{-1}=\left[\begin{array}{lll}b & E b & E^{2} b\end{array}\right]^{-1}=\left[\begin{array}{rrr}-1 & 0 & 0 \\ 0 & 0 & 1 \\ 0 & -2 & 0\end{array}\right]$.

Therefore, the desired state feedback gain $k^{\prime}$ is $\left[\begin{array}{lll}0 & -3 & -2\end{array}\right]$.

\section{Conclusion}

The generalized Ackermann's formula for the single input singular systems is proposed. In order to apply our formula, the controllable regular generalized system should be restricted equivalent transformed to its standard form by a matrix multiplication. Just like in the generalized Ackermann's formula, only the closed-loop characteristic polynomial is concerned. If $E$ is nonsingular then the gain matrix, $k^{\prime}$, can be computed from (11) of Theorem 1. If $E$ is singular then Theorem 2 will be used to compute $k^{\prime}$. In Theorem 2, both closed-loop and open-loop characteristic polynomials are needed. Although $k^{\prime}$ is computed based on the standard generalized system, if we use the same feedback law, $u(t)=-k^{\prime} x(t)+r(t)$, in the original regular system, the closed-loop poles are still the desired ones.

\section{References}

[1] J. Ackermann, Der Entwurf linearer Regelungssysteme im Zustandsram, Regelungstechnik 7 (1972) 297-300.

[2] A. Ailon, An approach for pole assignment in singular systems, IEEE Trans. Automat. Control 34 (1989) 889-893.

[3] V.A. Armentano, Eigenvalue placement for generalized linear systems, Systems Control Lett. 4 (1984) 199-202.

[4] F.R. Chang and H.C. Chen, The generalized CayleyHamilton theorem for standard pencils, Systems Control Lett. 18 (1992) 179-182.

[5] C.T. Chen, Control System Design (Saunders College, Fort Worth, TX, 1993). 
[6] J.D. Cobb, Feedback and pole-placement in descriptor variable systems, Internat. J. Control 33 (1981) $1135-1146$

[7] J.D. Cobb, Controllability, observability and duality in singular systems, IEEE Trans. Automat. Control 29 (1984) 1076-1082.

[8] G.F. Franklin, J.D. Powell and A. Emami-Naeini, Feedback Control of Dynamics Systems (Addison-Wesley, Reading, MA, 1991).

[9] F.R. Gantmacher, The Theory of Matrices, Vol. 2 (Chelsea, New York, 197.4).

[10] T. Kailath, Linear Systems (Prentice-Hall, Englewood Cliffs, NJ, 1980).
[11] R. Nikoukhan, A.S. Willsky and B.C. Levy, Boundaryvalue descriptor systems: well-posedness, reachability and observability, Internat. J. Control 46 (1987) 1715-1737.

[12] C.L. Philips and R.D. Harbor, Feedback Control Systems (Prentice-Hall, Englewood Cliffs, NJ, 1988).

[13] H.H. Rosenbrock, Structural properties of linear dynamical systems, Internat. J. Control 20 (1974) 191-202.

[14] G.C. Verghese, B.C. Levy and T. Kailath, A generalized state-space for singular systems, IEEE Trans. Automat. Control 26 (1981) 811-831.

[15] Y.Y. Wang, S.J. Shi and Z.J. Zhang, Pole placement and compensator design of generalized systems, Systems Control Lett. 8 (1987) 205-209. 